\title{
Effective Fractal Dimension in Algorithmic Information Theory
}

\author{
Elvira Mayordomo*
}

\section{Introduction}

Hausdorff dimension assigns a dimension value to each subset of an arbitrary metric space. In Euclidean space, this concept coincides with our intuition that smooth curves have dimension 1 and smooth surfaces have dimension 2, but from its introduction in 1918 [23] Hausdorff noted that many sets have noninteger dimension, what he called "fractional dimension". The development and applications of Fractal Geometry quickly outgrew the field of Geometry and spread through many other areas $[19,56,15,16,17,13,12,49]$. In the 1980s Tricot [73] and Sullivan [71] independently developed a dual of Hausdorff dimension called packing dimension that is now widely used.

In this paper we will focus on the use of fractal dimensions in the Cantor space of infinite sequences over a finite alphabet. The results obtained since the 1990's, and in particular the effectivizations of dimension that we will review in this paper, have introduced the powerful tools of fractal geometry into Computational Complexity and Information Theory.

In 2000 Lutz [45] proved a new characterization of Hausdorff dimension for the case of Cantor space that was based on gales. This characterization was the beginning of a whole range of effective versions of dimensions naturally based on bounding the computing power of the gale. Gales are a generalization of martingales which are strategies for betting on the successive bits of infinite binary sequences with fair payoffs. Martingales were introduced by Ville [74] in 1939 (also implicit in [38, 39]) and used by Schnorr [61, 62, 63, 64] in his work on randomness. In the 1990s, Ryabko [59, 60] and Staiger [69] proved several connections of Hausdorff dimension and martingales, that included relating the Hausdorff dimension of a set $X$ of binary sequences to the growth rates achievable by computable martingales betting on the sequences in $X$ (see section 4 for more details).

The introduction of resource-bounded dimension by Lutz [45] had the immediate motivation of overcoming the limitations of resource-bounded measure, a

* Departamento de Informática e Ingeniería de Sistemas, María de Luna 1, Universidad de Zaragoza, 50018 Zaragoza, SPAIN. elvira at unizar dot es. Research supported in part by Spanish Government MEC Project TIN 2005-08832-C03-02. 
generalization of classical Lebesgue measure, in the quantitative analysis of complexity classes [43]. The resulting concepts of effective dimension have turned out to be robust, since they have been shown to admit several equivalent definitions that relate them to well-studied concepts in computation, and they have proven very fruitful in investigating not only the structure of complexity classes but also in the modeling and analysis of sequence information and more recently, back in fractal geometry. See [30] for an updated bibliography on effective dimension.

There is a recent survey on the applications of effective dimension to the study of complexity classes by Hitchcock et al. [28]. The purpose of this paper will be centered in the Information Theory connections. In fact, as it could be suspected from earlier results by Ryabko [57, 58], Staiger [68, 69], and Cai and Hartmanis [3], effective dimensions have very clear interpretations in terms of information content or compressibility of a sequence. Considering different bounds on computing power that range from finite memory to constructibility, including time-bounded and space-bounded computations, effective dimensions capture what can be considered the inherent information content of a sequence in the corresponding setting. We will present in this paper all known characterizations of effective dimension that support this thesis.

We start by developing very general definitions of dimension, including an extension of scaled dimension to a general metric space. Scaled dimension allows a rescaling of dimension that can give more meaningful results for dimension 0 sets, for instance. It was introduced in [27] for the particular case of Cantor space with the usual metric, based on the uniform probability distribution. We think this more general definition will allow further insight into the interest of scaled dimension with different metrics.

Next we review the different notions of effective dimension, starting with finite-state dimension in which computation is restricted to finite-state devices. In this setting compression has been widely studied as a precursor of the LempelZiv algorithm [37]. Dai et al. [6] proved that finite-state dimension can be characterized in terms of information-lossless finite-state compressors, and Doty and Moser [10] remarked that a Kolmogorov-complexity like characterization is also possible from earlier results by Sheinwald, Lempel, and Ziv [67].

In section 4 we will develop constructive dimension that corresponds to the use of lower semicomputable strategies, and that has good properties inherited from the existence of a universal constructible semimeasure. Lutz introduced this notion in [46]. Athreya et al. [1] introduced the dual constructive strong dimension. We present a characterization of both notions of constructive dimension in terms of Kolmogorov complexity, present a correspondence principle stating that constructive dimension coincides with Hausdorff dimension for sufficiently simple sets, and summarize the main results. The open question of whether positive dimension sequences can substitute Martin-Löf random sequences as the randomness source of a computation has received recent attention from different areas. We present the main known results here and refer the reader to [11] and [51] for more information (these two references use the term "effective dimension" for Lutz's constructive dimension). 
Our last section concerns resource-bounds on time and space. Polynomialspace bounded dimension has been well studied in terms of information content [25], but polynomial-time dimension seems harder to grasp. We know very little about time-bounded Kolmogorov complexity, but a compressibility characterization of polynomial-time dimension has been obtained in [41] via polynomial-time compression algorithms. We should consider polynomial-time dimension as an interesting alternative to time-bounded Kolmogorov Complexity expecting that we can import robustness properties from fractal dimension.

There are many related topics we chose not to cover in this paper, mainly due to lack of space for a proper development. Let us mention very interesting recent results on effective dimension on Euclidean space ([21], [47]) that would require a paper on its own.

\section{Fractal dimensions and gale characterizations}

In this section we first review the classical Hausdorff and packing dimensions and then we introduce scaled dimension for a general metric space. We present the characterizations of these notions in terms of gales for the case of Cantor space. This characterization is crucial in the definition of effective dimensions that we will introduce in the rest of the paper.

\subsection{Hausdorff and packing dimensions}

Let $\rho$ be a metric on a set $\mathcal{X}$. We use the following standard terminology. The diameter of a set $X \subseteq \mathcal{X}$ is $\operatorname{diam}(X)=\sup \{\rho(x, y) \mid x, y \in X\}$ (which may be $\infty)$. For each $x \in \mathcal{X}$ and $r \in \mathbb{R}$, the closed ball of radius $r$ about $x$ is the set $B(x, r)=\{y \in \mathcal{X} \mid \rho(y, x) \leq r\}$, and the open ball of radius $r$ about $x$ is the set $B^{o}(x, r)=\{y \in \mathcal{X} \mid \rho(y, x)<r\}$. A ball is any set of the form $B(x, r)$ or $B^{o}(x, r)$. A ball $B$ is centered in a set $X \subseteq \mathcal{X}$ if $B=B(x, r)$ or $B=B^{o}(x, r)$ for some $x \in X$ and $r \geq 0$.

For each $\delta>0$, we let $\mathcal{C}_{\delta}$ be the set of all countable collections $\mathcal{B}$ of balls such that $\operatorname{diam}(B) \leq \delta$ for all $B \in \mathcal{B}$, and we let $\mathcal{D}_{\delta}$ be the set of all $\mathcal{B} \in \mathcal{C}_{\delta}$ such that the balls in $\mathcal{B}$ are pairwise disjoint. For each $X \subseteq \mathcal{X}$ and $\delta>0$, we define the sets

$$
\begin{aligned}
& \mathcal{H}_{\delta}(X)=\left\{\mathcal{B} \in \mathcal{C}_{\delta} \mid X \subseteq \bigcup_{B \in \mathcal{B}} B\right\} \\
& \mathcal{P}_{\delta}(X)=\left\{\mathcal{B} \in \mathcal{D}_{\delta} \mid(\forall B \in \mathcal{B}) B \text { is centered in } X\right\} .
\end{aligned}
$$

If $\mathcal{B} \in \mathcal{H}_{\delta}(X)$, then we call $\mathcal{B}$ a $\delta$-cover of $X$. If $\mathcal{B} \in \mathcal{P}_{\delta}(X)$, then we call $\mathcal{B}$ a $\delta$-packing of $X$. For $X \subseteq \mathcal{X}, \delta>0$ and $s \geq 0$, we define the quantities

$$
\begin{aligned}
H_{\delta}^{s}(X) & =\inf _{\mathcal{B} \in \mathcal{H}_{\delta}(X)} \sum_{B \in \mathcal{B}} \operatorname{diam}(B)^{s} \\
P_{\delta}^{s}(X) & =\sup _{\mathcal{B} \in \mathcal{P}_{\delta}(X)} \sum_{B \in \mathcal{B}} \operatorname{diam}(B)^{s} .
\end{aligned}
$$


Since $H_{\delta}^{s}(X)$ and $P_{\delta}^{s}(X)$ are monotone as $\delta \rightarrow 0$, the limits

$$
\begin{aligned}
H^{s}(X) & =\lim _{\delta \rightarrow 0} H_{\delta}^{s}(X), \\
P_{0}^{s}(X) & =\lim _{\delta \rightarrow 0} P_{\delta}^{s}(X)
\end{aligned}
$$

exist, though they may be infinite. Let

$$
P^{s}(X)=\inf \left\{\sum_{i=0}^{\infty} P_{0}^{s}\left(X_{i}\right) \mid X \subseteq \bigcup_{i=0}^{\infty} X_{i}\right\} .
$$

It is routine to verify that the set functions $H^{s}$ and $P^{s}$ are outer measures [16]. The quantities $H^{s}(X)$ and $P^{s}(X)$ - which may be infinite - are called the $s$-dimensional Hausdorff (outer) ball measure and the $s$-dimensional packing (outer) ball measure of $X$, respectively. The optimization (2.1) over all countable partitions of $X$ is needed because the set function $P_{0}^{s}$ is not an outer measure.

Definition. Let $\rho$ be a metric on a set $\mathcal{X}$, and let $X \subseteq \mathcal{X}$.

1. (Hausdorff [23]). The Hausdorff dimension of $X$ with respect to $\rho$ is

$$
\operatorname{dim}^{(\rho)}(X)=\inf \left\{s \in[0, \infty) \mid H^{s}(X)=0\right\} .
$$

2. (Tricot [73], Sullivan [71]). The packing dimension of $X$ with respect to $\rho$ is

$$
\operatorname{Dim}^{(\rho)}(X)=\inf \left\{s \in[0, \infty) \mid P^{s}(X)=0\right\} .
$$

When $\mathcal{X}$ is a Euclidean space $\mathbb{R}^{n}$ and $\rho$ is the usual Euclidean metric on $\mathbb{R}^{n}, \operatorname{dim}^{(\rho)}$ and $\operatorname{Dim}^{(\rho)}$ are the ordinary Hausdorff and packing dimensions, also denoted by $\operatorname{dim}_{H}$ and $\operatorname{dim}_{P}$, respectively.

\subsection{Scaled dimensions}

This subsection introduces the notion of scaled dimension for a general metric space. Our treatment is based on [27], that presents only the case of Cantor space and uses directly gales in the definition.

The notions of Hausdorff and packing dimensions introduced above depend on the expression "diam $(B)^{s "}$ that is used both in $s$-Haussdorff and $s$-packing measures (see definitions of $H_{\delta}^{s}(X)$ and $P_{\delta}^{s}(X)$ above). Here we consider alternative functions on $s$ and the diameter.

Definition. A scale is a function $h(x, s), h:[0, \infty) \times[0, \infty) \rightarrow[0, \infty)$, with the following two properties:

1. For every $s \in[0, \infty), h\left({ }_{-}, s\right)$ is nondecreasing.

2. For every $s, \epsilon \in[0, \infty), \lim _{x \rightarrow 0} \frac{h(x, s+\epsilon)}{h(x, s)}=0$. 
From each scale $h$ we define scaled Hausdorff and packing measures generalizing the definitions in subsection 2.1 .

For $X \subseteq \mathcal{X}, \delta>0$ and $s \geq 0$, we define the quantities

$$
\begin{aligned}
S H_{\delta}^{h, s}(X) & =\inf _{\mathcal{B} \in \mathcal{H}_{\delta}(X)} \sum_{B \in \mathcal{B}} h(\operatorname{diam}(B), s), \\
S P_{\delta}^{h, s}(X) & =\sup _{\mathcal{B} \in \mathcal{P}_{\delta}(X)} \sum_{B \in \mathcal{B}} h(\operatorname{diam}(B), s) .
\end{aligned}
$$

Since $S H_{\delta}^{s}(X)$ and $S P_{\delta}^{s}(X)$ are monotone as $\delta \rightarrow 0$, the limits

$$
\begin{aligned}
& S H^{h, s}(X)=\lim _{\delta \rightarrow 0} S H_{\delta}^{h, s}(X), \\
& S P_{0}^{h, s}(X)=\lim _{\delta \rightarrow 0} S P_{\delta}^{h, s}(X)
\end{aligned}
$$

exist, though they may be infinite. Let

$$
S P^{h, s}(X)=\inf \left\{\sum_{i=0}^{\infty} S P_{0}^{h, s}\left(X_{i}\right) \mid X \subseteq \bigcup_{i=0}^{\infty} X_{i}\right\} .
$$

In this case it is also routine to verify that the set functions $S H^{h, s}$ and $S P^{h, s}$ are outer measures. The optimization (2.2) over all countable partitions of $X$ is needed because the set function $S P_{0}^{h, s}$ is not an outer measure.

Rogers introduced in [56] the generalized notion of Hausdorff measure using a function $f(\operatorname{diam}(B))$ in the place of $\operatorname{diam}(B)^{s}$ in the definition of Hausdorff measure. More recently Roger's approach was revisited by Reimann and Stephan [55] in the context of algorithmic randomness. In those references the authors didn't consider dependence on a second parameter $s$ or a dimension concept in this context.

Our first property is that for each $X \subseteq \mathcal{X}$ there is at most one $s$ for which $0<S H^{h, s}(X)<\infty$.

Proposition 2.1 Let $X \subseteq \mathcal{X}$, let $h$ be a scale, and $s \in[0, \infty)$.

1. If $0<S H^{h, s}(X)<\infty$ then for every $\epsilon>0, S H^{h, s+\epsilon}(X)=0$.

2. If $0<S P^{h, s}(X)<\infty$ then for every $\epsilon>0, S P^{h, s+\epsilon}(X)=0$.

Proof. The property follows from the fact that $\lim _{x \rightarrow 0} \frac{h(x, s+\epsilon)}{h(x, s)}=0$, in the definition of scale.

Definition. Let $\rho$ be a metric on a set $\mathcal{X}$, let $X \subseteq \mathcal{X}$, and let $h$ be a scale.

1. The $h$-scaled dimension of $X$ with respect to $\rho$ is

$$
\operatorname{dim}^{(h),(\rho)}(X)=\inf \left\{s \in[0, \infty) \mid S H^{h, s}(X)=0\right\} .
$$

2. The $h$-scaled packing dimension of $X$ with respect to $\rho$ is

$$
\operatorname{Dim}^{(h),(\rho)}(X)=\inf \left\{s \in[0, \infty) \mid S P^{h, s}(X)=0\right\} .
$$


The basic properties of scaled-dimensions are monotonicity and countable stability, that also hold for Hausdorff and packing dimension [16].

Proposition 2.2 Let $h$ be a scale.

1. For every $x \in \mathcal{X}, \operatorname{Dim}^{(h),(\rho)}(\{x\})=\operatorname{dim}^{(h),(\rho)}(\{x\})=0$.

2. For every $X \subseteq \mathcal{X}, 0 \leq \operatorname{dim}^{(h),(\rho)}(X) \leq \operatorname{Dim}^{(h),(\rho)}(X)$.

3. Let $X_{i} \subseteq \mathcal{X}$ for each $i \in \mathbb{N}$,

$$
\begin{gathered}
\operatorname{dim}^{(h),(\rho)}\left(\cup_{i} X_{i}\right)=\sup _{i} \operatorname{dim}^{(h),(\rho)}\left(X_{i}\right), \text { and } \\
\operatorname{Dim}^{(h),(\rho)}\left(\cup_{i} X_{i}\right)=\sup _{i} \operatorname{Dim}^{(h),(\rho)}\left(X_{i}\right) .
\end{gathered}
$$

4. For every $X, Y \subseteq \mathcal{X}$ with $X \subseteq Y$,

$$
\operatorname{dim}^{(h),(\rho)}(X) \leq \operatorname{dim}^{(h),(\rho)}(Y) .
$$

and

$$
\operatorname{Dim}^{(h),(\rho)}(X) \leq \operatorname{Dim}^{(h),(\rho)}(Y)
$$

In particular, every countable set has zero scaled-dimension for any scale.

Notice that for $h_{0}(x, s)=x^{s}, \operatorname{dim}^{\left(h_{0}\right),(\rho)}(X)=\operatorname{dim}^{(\rho)}(X)$ and $\operatorname{Dim}^{\left(h_{0}\right),(\rho)}(X)=$ $\operatorname{Dim}^{(\rho)}(X)$.

We can compare the scaled dimensions for different scales.

Proposition 2.3 Let $h, h^{\prime}$ be scales such that $h(x, s) \leq h^{\prime}(x, s)$ for every $s$ and for every $x \in[0, \epsilon)$, where $\epsilon>0$ may depend on $s$. Then for every $X \subseteq \mathcal{X}$,

$$
\operatorname{dim}^{(h),(\rho)}(X) \leq \operatorname{dim}^{\left(h^{\prime}\right),(\rho)}(X)
$$

and

$$
\operatorname{Dim}^{(h),(\rho)}(X) \leq \operatorname{Dim}^{\left(h^{\prime}\right),(\rho)}(X) .
$$

Proof. The property follows from the definition of scaled Hausdorff and packing measures.

The next property concerns the scaled dimension of the whole space.

Proposition 2.4 Let $\mathcal{X}$ be a metric space such that $0<H^{\operatorname{dim}(\mathcal{X})}(\mathcal{X})<\infty$. Let $h$ be a scale, let $s \in[0, \infty)$.

If $h(x, s)=\Omega\left(x^{\operatorname{dim}(\mathcal{X})}\right)$ then $\operatorname{dim}^{(h)}(\mathcal{X}) \geq s$.

If $h(x, s)=O\left(x^{\operatorname{dim}(\mathcal{X})}\right)$ then $\operatorname{dim}^{(h)}(\mathcal{X}) \leq s$.

Lutz et al. consider in [27] the following scales, that are useful for dimension values up to 1 .

Definition. For every $x \in[0, \infty), s \in[0, \infty)$ we define

1. $h_{0}(x, s)=x^{s}$. 
2. For each $i \geq 0$,

$$
\begin{array}{ll}
h_{i+1}(x, s)=2^{-\frac{1}{h_{i}(-1 / \log (x), s)}} & \text { if } x \leq 1 / 2, s<1 \\
h_{i+1}(x, s)=2^{-1 / h_{i}(1, s)} & \text { if } x>1 / 2, s<1 \\
h_{i+1}(x, s)=x^{s} & \text { if } s \geq 1
\end{array}
$$

3. For each $i>0$,

$$
h_{-i}(x, s)= \begin{cases}x / h_{i}(x, 1-s) & \text { if } s<1 \\ x^{s} & \text { if } s \geq 1\end{cases}
$$

For $s<1$, the above defined scales are below the inverse of the logarithm, and for every $k, h_{k}$ is asymptotically below $h_{k+1}$. This provides a fine family of scales that for instance can be used to distinguish different circuit size rates [27].

Proposition 2.5 For every $k \in \mathbb{Z}$, the above defined $h_{k}$ is a scale.

Notation. For each $k \in \mathbb{Z}$, we denote $\operatorname{dim}^{\left(h_{k}\right),(\rho)}(X)$ as $\operatorname{dim}^{(k),(\rho)}(X)$ and $\operatorname{Dim}^{\left(h_{k}\right),(\rho)}(X)$ as $\operatorname{Dim}^{(k),(\rho)}(X)$.

The relationship between scales $h_{k}$ is the following.

Proposition 2.6 Let $k \in \mathbb{Z}$. Then for every $X \subseteq \mathcal{X}$,

$$
\operatorname{dim}^{(k),(\rho)}(X) \leq \operatorname{dim}^{(k+1),(\rho)}(X)
$$

and

$$
\operatorname{Dim}^{(k),(\rho)}(X) \leq \operatorname{Dim}^{(k+1),(\rho)}(X) .
$$

\subsection{Gale characterizations}

We now focus our attention on sequence spaces. Let $\Sigma$ be a finite alphabet with $|\Sigma| \geq 2$. We will consider the following metric on $\Sigma^{\infty}$

$$
\rho(S, T)=\inf \left\{|\Sigma|^{-|w|} \mid w \sqsubseteq S \text { and } w \sqsubseteq T\right\}
$$

for all $S, T \in \Sigma^{\infty}$.

We fix the above $\rho$ and denote $\operatorname{dim}^{(\rho)}(X)$ and $\operatorname{Dim}^{(\rho)}(X)$ as $\operatorname{dim}(X)$ and $\operatorname{Dim}(X)$, for $X \subseteq \Sigma^{\infty}$. Similarly for scaled dimension we use $\operatorname{dim}^{(h)}(X)$ and $\operatorname{Dim}^{(h)}(X)$ for $\operatorname{dim}^{(h),(\rho)}(X), \operatorname{Dim}^{(h),(\rho)}(X)$. Recently Lutz and Mayordomo have considered alternative metrics on $\Sigma^{\infty}$ with interesting applications to dimension in Euclidean space [47].

Lutz [45] characterized Hausdorff dimension in terms of gales, presented next.

Definition. [45] Let $\Sigma$ be a finite alphabet with $|\Sigma| \geq 2$ and let $s \in[0, \infty)$. 
1. An $s$-gale is a function $d: \Sigma^{*} \rightarrow[0, \infty)$ that satisfies the condition

$$
d(w)=|\Sigma|^{-s} \sum_{a \in \Sigma} d(w a)
$$

for all $w \in \Sigma^{*}$.

2. A martingale is a 1-gale.

In fact Lutz [45] considered also supergales, that are functions for which equality (2.3) is substituted by the inequality

$$
d(w) \geq|\Sigma|^{-s} \sum_{a \in \Sigma} d(w a) .
$$

Supergales give additional flexibility and in most interesting cases can be substituted by gales in the definitions and characterizations of different dimensions and effective dimensions. For the sake of readability we will restrict to gales in this paper.

The following observation shows how gales are affected by variation of the parameter $s$.

Observation $2.7[46]$. Let $s, s^{\prime} \in[0, \infty)$ and let $d, d^{\prime}: \Sigma^{*} \rightarrow[0, \infty)$. Assume that

$$
d(w)|\Sigma|^{-s|w|}=d^{\prime}(w)|\Sigma|^{-s^{\prime}|w|}
$$

holds for all $w \in \Sigma^{*}$. Then $d$ is an s-gale if and only if $d^{\prime}$ is an $s^{\prime}$-gale.

For example, a function $d: \Sigma^{*} \rightarrow[0, \infty)$ is an $s$-gale if and only if the function $d^{\prime}: \Sigma^{*} \rightarrow[0, \infty)$ defined by $d^{\prime}(w)=|\Sigma|^{1-s|w|} d(w)$ is a martingale.

Martingales were introduced by Lévy [39] and Ville [74]. They have been used extensively by Schnorr [62,63,65] and others in investigations of randomness, by Lutz $[42,44]$ and others in the development of resource-bounded measure, and by Ryabko [60] and Staiger [69] regarding exponents of increase. Gales are a convenient generalization of martingales introduced by Lutz $[45,46]$ in the development of effective fractal dimensions.

Intuitively, an $s$-gale $d$ is a strategy for betting on the successive symbols in a sequence $S \in \Sigma^{\infty}$. We regard the value $d(w)$ as the amount of money that a gambler using the strategy $d$ will have after betting on the symbols in $w$, is $w$ is a prefix of $S$. If $s=1$, then the $s$-gale identity (2.3) ensures that the payoffs are fair in the sense that the conditional expected value of the gambler's capital after the symbol following $w$, given that $w$ has occurred, is precisely $d(w)$, the gambler's capital after $w$. If $s<1$, then (2.3) says that the payoffs are less than fair. If $s>1$, then (2.3) says that the payoffs are more than fair. Clearly, the smaller $s$ is, the more hostile the betting environment is.

There are two important notions of success for a gale.

Definition. Let $d$ be an $s$-gale, where $s \in[0, \infty)$, and let $S \in \Sigma^{\infty}$.

1. We say that $d$ succeeds on $S$, and we write $S \in S^{\infty}[d]$, if $\lim \sup _{t \rightarrow \infty} d(S[0 . . t-1])=\infty$. 
2. We say that $d$ succeeds strongly on $S$, and we write $S \in S_{\mathrm{str}}^{\infty}[d]$, if $\liminf _{t \rightarrow \infty} d(S[0 . . t-1])=\infty$.

The following theorem gives useful characterizations of the classical Hausdorff and packing dimensions on sequence spaces. The Hausdorff dimension part was proven by Lutz [45] and the packing dimension part was proven by Athreya et al. in [1].

Theorem 2.8 ([45] and [1]) For all $X \subseteq \Sigma^{\infty}$,

$$
\operatorname{dim}(X)=\inf \left\{s \in[0, \infty) \mid \text { there is an s-gale } d \text { with } X \subseteq S^{\infty}[d]\right\},
$$

and

$$
\operatorname{Dim}(X)=\inf \left\{s \in[0, \infty) \mid \text { there is an s-gale } d \text { with } X \subseteq S_{\mathrm{str}}^{\infty}[d]\right\} .
$$

The effectivization of both Hausdorff and packing (or strong) dimension will be based on Theorem 2.8. By restricting the set of gales that are allowed to different classes of computable gales, we will obtain effective versions of dimension that will be meaningful in different subclasses of $\Sigma^{\infty}$. This will be developed in the following sections.

Eggleston [14] proved the following classical result on the Hausdorff dimension of a set of sequences with a fixed asymptotic frequency.

The frequency of a nonempty binary string $w \in\{0,1\}^{*}$ is the ratio freq $(w)=$ $\frac{\#(1, w)}{|w|}$, where \#(b,w) denotes the number of occurrences of the bit $b$ in $w$. For each $\alpha \in[0,1]$, we define the set

$$
\operatorname{FREQ}(\alpha)=\left\{S \in\{0,1\}^{\infty} \mid \lim _{n \rightarrow \infty} \operatorname{freq}(S[0 . . n-1])=\alpha\right\} .
$$

The binary Shannon entropy function $\mathcal{H}:[0,1] \rightarrow[0,1]$ is defined as $\mathcal{H}(x)=$ $x \log \frac{1}{x}+(1-x) \log \frac{1}{1-x}$, with $\mathcal{H}(0)=\mathcal{H}(1)=0$.

Theorem 2.9 [14] For each real number $\alpha \in[0,1]$,

$$
\operatorname{dim}_{\mathrm{H}}(\operatorname{FREQ}(\alpha))=\mathcal{H}(\alpha) .
$$

We will reformulate this last result in the contexts of the dimensions defined in sections 3,4 , and 5 .

We finish this section with the fact that scaled-dimension in $\Sigma^{\infty}$ admits a similar characterization.

The notion of scaled-gales is introduced in [27].

Definition. Let $\Sigma$ be a finite alphabet with $|\Sigma| \geq 2$, let $h$ be a scale and let $s \in[0, \infty)$. An $h$-scaled $s$-gale (briefly, an $s^{(h)}$-gale) is a function $d: \Sigma^{*} \rightarrow[0, \infty)$ that satisfies the condition

$$
h\left(|\Sigma|^{-|w|}, s\right) d(w)=h\left(|\Sigma|^{-(|w|+1)}, s\right) \sum_{a \in \Sigma} d(w a)
$$

for all $w \in \Sigma^{*}$.

Notice that our definition of gale (Definition 2.3) corresponds to the scale $h_{0}(x, s)=x^{s}$, so an $s^{\left(h_{0}\right)}$-gale is just an $s$-gale. 
Observation 2.10 Let $h, h^{\prime}$ be scales, let $s, s^{\prime} \in[0, \infty)$ and let $d: \Sigma^{*} \rightarrow[0, \infty)$. $d$ is an $s^{(h)}$-gale if and only if

$$
d^{\prime}(w)=\frac{h\left(|\Sigma|^{-|w|}, s\right)}{h^{\prime}\left(|\Sigma|^{-|w|}, s^{\prime}\right)} d(w)
$$

is an $s^{\left(h^{\prime}\right)}$-gale.

Success and strong success are defined as follows.

Definition. Let $d$ be an $s^{(h)}$-gale, where $h$ is a scale, $s \in[0, \infty)$, and let $S \in \Sigma^{\infty}$. We say that $d$ succeeds on $S$, and we write $S \in S^{\infty}[d]$, if

$\lim \sup _{t \rightarrow \infty} d(S[0 . . t-1])=\infty$. We say that $d$ succeeds strongly on $S$, and we write $S \in S_{\mathrm{str}}^{\infty}[d]$, if

$\liminf _{t \rightarrow \infty} d(S[0 . . t-1])=\infty$.

Lutz et al. defined scaled-dimension in Cantor space directly using gales in [27]. Here we introduced a more general concept of scaled-dimension for any metric space and now characterize the Cantor space case.

Theorem 2.11 For all $X \subseteq \Sigma^{\infty}$,

$$
\operatorname{dim}^{(h)}(X)=\inf \left\{s \in[0, \infty) \mid \text { there is an } s^{(h)} \text {-gale } d \text { with } X \subseteq S^{\infty}[d]\right\},
$$

and

$$
\operatorname{Dim}^{(h)}(X)=\inf \left\{s \in[0, \infty) \mid \text { there is an } s^{(h)} \text {-gale } d \text { with } X \subseteq S_{\mathrm{str}}^{\infty}[d]\right\} .
$$

For space reasons, we prefer not to include a full proof of Theorem 2.11 here. The proof can be done by nontrivially adapting the proofs of both parts of Theorem 2.8 that can be found in [45] and [1], respectively.

Our last property identifies the scales for which Cantor space has dimension 1 .

Proposition 2.12 Let $h$ be a scale such that $h(x, s)=\Omega(x)$ for every $s<1$ and $h(x, s)=O(x)$ for every $s>1$. Then

$$
\operatorname{dim}^{(h)}\left(\Sigma^{\infty}\right)=\operatorname{Dim}^{(h)}\left(\Sigma^{\infty}\right)=1 .
$$

For every $k \in \mathbb{Z}$,

$$
\operatorname{dim}^{(k)}\left(\Sigma^{\infty}\right)=\operatorname{Dim}^{(k)}\left(\Sigma^{\infty}\right)=1 .
$$

Proof. The property follows from Proposition 2.4.

\subsection{Effective dimensions}

We are mainly interested in subsets of sequences that have some computability or partial computability property, which implies that we will deal with countable sets. Since a countable set of sequences has dimension 0, the classical definitions of (scaled)-Hausdorff and packing dimensions are not useful in this context. 
The gale characterizations in Theorems 2.11 and 2.8 provide a natural way to generalize them as follows.

Definition. Let $\Gamma$ be a class of functions. Let $X \subseteq \Sigma^{\infty}$, the $\Gamma$-dimension of $X$ is

$$
\operatorname{dim}_{\Gamma}(X)=\inf \left\{s \in[0, \infty) \mid \text { there is an } s \text {-gale } d \in \Gamma \text { with } X \subseteq S^{\infty}[d]\right\},
$$

and the $\Gamma$-strong dimension of $X$ is

$$
\operatorname{Dim}_{\Gamma}(X)=\inf \left\{s \in[0, \infty) \mid \text { there is an s-gale } d \in \Gamma \text { with } X \subseteq S_{\text {str }}^{\infty}[d]\right\} .
$$

In the rest of the paper we will use different classes $\Gamma$, ranging from constructive to finite state computable functions, and investigate the properties of the corresponding $\Gamma$-dimensions inside different sequence sets. The existence of correspondence principles, introduced later on, will also imply that the effective dimension coincides with the classical Hausdorff dimension on sufficiently simple sets.

For scaled-dimensions it is convenient that the scale itself is "computable" inside $\Gamma$ in order to obtain meaningful results. Given a scale $h$, we will say that $h$ is $\Gamma$-computable if the function $d h: \mathbb{N} \times[0, \infty) \rightarrow[0, \infty), d h(k, s)=$ $h\left(|\Sigma|^{-k-1}, s\right) / h\left(|\Sigma|^{-k}, s\right)$ is in $\Gamma$. The definitions of $\operatorname{dim}_{\Gamma}^{(h)}$ and $\operatorname{Dim}_{\Gamma}^{(h)}$ are similar to those of $\Gamma$-dimensions, but use $s^{(h)}$-gales in $\Gamma$.

\section{Finite-State Dimension}

Our first effectivization of Hausdorff dimension will be the most restrictive of those presented here, we will go all the way to the level of finite-state computation. In this section we use gales computed by finite-state gamblers to develop the finite-state dimensions of sets of infinite sequences and individual infinite sequences. Finite-state dimension was introduced by Dai et al. in [6] and its dual, strong finite-state dimension, is from [1]. The definition has proven to be robust because it has been shown to admit equivalent definitions in terms of information-lossless finite-state compressors [6, 1], finite-state decompression [10], finite-state predictors in the log-loss model [26, 1], and block-entropy rates [2]. In each case, the definitions of $\operatorname{dim}_{\mathrm{FS}}(S)$ and $\operatorname{Dim}_{\mathrm{FS}}(S)$ are exactly dual, differing only that a limit inferior appears in one definition where a limit superior appears in the other. These two finite-state dimensions are thus, like their counterparts in fractal geometry, robust quantities and not artifacts of a particular definition. In addition, the sequences $S$ satisfying $\operatorname{dim}_{\mathrm{FS}}(S)=1$ are precisely the normal sequences ([2], also follows from [66]).

In this section we present finite-state dimension and its characterizations and summarize the main results on Eggleston theorem, existence of low complexity sequences of any dimension, invariance of finite-state dimension under arithmetic operations with rational numbers, and base dependence of the dimensions. 
We start by introducing the concept of finite-state gambler that is used to develop finite-state dimension. Intuitively, a finite-state gambler is a finite-state device that places a bet on each of the successive symbols of its input sequence. Bets are required to be rational numbers in $\mathbf{B}=\mathbb{Q} \cap[0,1]$.

Definition. A finite-state gambler $(F S G)$ is a 4 -tuple $G=\left(Q, \delta, \beta, q_{0}\right)$, where

- $Q$ is a nonempty, finite set of states,

- $\delta: Q \times \Sigma \rightarrow Q$ is the transition function,

- $\beta: Q \times \Sigma \rightarrow \mathbf{B}$ is the betting function, with $\sum_{a \in \Sigma} \beta(q, a)=1$ for every $q \in Q$, and

- $q_{0} \in Q$ is the initial state.

Dai et al. [6], consider an equivalent model, the $k$-account finite-state gambler, in which the capital is divided into $k$ separate accounts for a fixed $k$. This model allows simpler descriptions and a smaller number of states in the gambler definitions.

Our model of finite-state gambling that has been considered (in essentially equivalent form) by Schnorr and Stimm [66], Feder [18], and others.

Intuitively, if a FSG $G=\left(Q, \delta, \beta, q_{0}\right)$ is in state $q \in Q$ and its current capital is $c \in(\mathbb{Q} \cap[0, \infty))$, then it places the bet $\beta(q, a) \in \mathbf{B}$ on each possible value of the next symbol. If the payoffs are fair, then after this bet $G$ will be in state $\delta(q, a)$ and it will have capital $|\Sigma| c \beta(q, a)$.

This suggests the following definition.

Definition. [6] Let $G=\left(Q, \delta, \beta, q_{0}\right)$ be a finite-state gambler.

1. The martingale of $G$ is the function $d_{G}: \Sigma^{*} \rightarrow[0, \infty)$ defined by the recursion

$$
\begin{aligned}
& d_{G}(\lambda)=1, \\
& d_{G}(w a)=|\Sigma| d_{G, i}(w) \beta(q, a)
\end{aligned}
$$

for all $w \in \Sigma^{*}$ and $a \in \Sigma$.

2. For $s \in[0, \infty)$, the $s$-gale of an FSG $G$ is the function $d_{G}^{(s)}: \Sigma^{*} \rightarrow[0, \infty)$ defined by $d_{G}^{(s)}(w)=|\Sigma|^{(s-1)|w|} d_{G}(w)$ for all $w \in \Sigma^{*}$. In particular, note that $d_{G}^{(1)}=d_{G}$.

3. For $s \in[0, \infty)$, a finite-state $s$-gale is an $s$-gale $d$ for which there exists an FSG $G$ such that $d_{G}^{(s)}=d$.

We now use finite-state gales to define finite-state dimension.

Definition. $[6,1]$ Let $X \subseteq \Sigma^{\infty}$.

1. The finite-state dimension of set $X$ is

$\operatorname{dim}_{\mathrm{FS}}(X)=\inf \left\{s \in[0, \infty) \mid\right.$ there is a finite-state $s$-gale $d$ with $\left.X \subseteq S^{\infty}[d]\right\}$ 
2. The strong finite-state dimension of set $X$ is

$\operatorname{Dim}_{\mathrm{FS}}(X)=\inf \left\{s \in[0, \infty) \mid\right.$ there is a finite-state $s$-gale $d$ with $\left.X \subseteq S_{\mathrm{str}}^{\infty}[d]\right\}$

3. The finite-state dimension and strong finite-state dimension of a sequence $S \in \Sigma^{\infty}$ are $\operatorname{dim}_{\mathrm{FS}}(S)=\operatorname{dim}_{\mathrm{FS}}(\{S\})$ and $\operatorname{Dim}_{\mathrm{FS}}(S)=\operatorname{Dim}_{\mathrm{FS}}(\{S\})$

In general, $\operatorname{dim}_{\mathrm{FS}}(X)$ and $\operatorname{Dim}_{\mathrm{FS}}(X)$ are real numbers satisfying $0 \leq \operatorname{dim}_{\mathrm{H}}(X)$ $\leq \operatorname{dim}_{F S}(X) \leq \operatorname{Dim}_{F S}(X) \leq 1$ and $\operatorname{Dim}(X) \leq \operatorname{Dim}_{F S}(X)$. Finite-state dimension has a finite stability property.

Theorem 3.1 [6] For all $X, Y \subseteq \Sigma^{\infty}$,

$$
\operatorname{dim}_{F S}(X \cup Y)=\max \left\{\operatorname{dim}_{F S}(X), \operatorname{dim}_{F S}(Y)\right\} .
$$

The proof of basic properties such as this theorem in [6] benefits greatly from the use of multiple account FSGs, since the equivalence of multiple accounts and our 1-account FSG seems to require an exponential blowup of states.

The main result in this section is that we can characterize the finite-state dimensions of individual sequences in terms of finite-state compressibility. We first recall the definition of an information-lossless finite-state compressor. (This idea is due to Huffman [34]. Further exposition may be found in [35] or [36].)

Definition. A finite-state transducer is a 4-tuple $C=\left(Q, \delta, \nu, q_{0}\right)$, where $Q$ is a nonempty, finite set of states, $\delta: Q \times \Sigma \rightarrow Q$ is the transition function, $\nu: Q \times \Sigma \rightarrow \Sigma^{*}$ is the output function, and $q_{0} \in Q$ is the initial state.

For $q \in Q$ and $w \in \Sigma^{*}$, we define the output from state $q$ on input $w$ to be the string $\nu(q, w)$ defined by the recursion

$$
\begin{aligned}
& \nu(q, \lambda)=\lambda \\
& \nu(q, w a)=\nu(q, w) \nu(\delta(q, w), a)
\end{aligned}
$$

for all $w \in \Sigma^{*}$ and $a \in \Sigma$. We then define the output of $C$ on input $w \in \Sigma^{*}$ to be the string $C(w)=\nu\left(q_{0}, w\right)$.

Definition. An information-lossless finite-state compressor (ILFSC) is a finitestate transducer $C=\left(Q, \delta, \nu, q_{0}\right)$ such that the function $f: \Sigma^{*} \rightarrow \Sigma^{*} \times Q$, $f(w)=(C(w), \delta(w))$ is one-to-one.

That is, an ILFSC is an transducer whose input can be reconstructed from the output and final state reached on that input.

Intuitively, $C$ compresses a string $w$ if $|C(w)|$ is significantly less than $|w|$. Of course, if $C$ is IL, then not all strings can be compressed. Our interest here is in the degree (if any) to which the prefixes of a given sequence $S \in \Sigma^{\infty}$ can be compressed by an ILFSC. We will consider the cases of infinitely often (i.o.) and almost everywhere (a.e.) compression ratio.

\section{Definition.}

1. If $C$ is an ILFSC and $S \in \Sigma^{\infty}$, then the a.e. compression ratio of $C$ on $S$ is

$$
\rho_{C}(S)=\liminf _{n \rightarrow \infty} \frac{|C(S[0 . . n-1])|}{n} .
$$


2. The finite-state a.e. compression ratio of a sequence $S \in \Sigma^{\infty}$ is

$$
\rho_{\mathrm{FS}}(S)=\inf \left\{\rho_{C}(S) \mid C \text { is an } \operatorname{ILFSC}\right\} .
$$

3. If $C$ is an ILFSC and $S \in \Sigma^{\infty}$, then the a.e. compression ratio of $C$ on $S$ is

$$
R_{C}(S)=\limsup _{n \rightarrow \infty} \frac{|C(S[0 . . n-1])|}{n} .
$$

4. The finite-state i.o. compression ratio of a sequence $S \in \Sigma^{\infty}$ is

$$
R_{\mathrm{FS}}(S)=\inf \left\{R_{C}(S) \mid C \text { is an ILFSC }\right\} .
$$

The following theorem says that finite-state dimension and finite-state compressibility are one and the same for individual sequences.

Theorem 3.2 [6, 1] For all $S \in \Sigma^{\infty}$,

$$
\operatorname{dim}_{\mathrm{FS}}(S)=\rho_{\mathrm{FS}}(S) .
$$

and

$$
\operatorname{Dim}_{\mathrm{FS}}(S)=R_{\mathrm{FS}}(S)
$$

Doty and Moser [10] remarked that finite-state dimension can be characterized in terms of decompression by finite-state transducers based on earlier results by Sheinwald, Lempel, and Ziv [67]. Notice that in this case finite-state machines are not required to be information lossless.

Theorem 3.3 [10] For all $S \in \Sigma^{\infty}$,

$$
\operatorname{dim}_{\mathrm{FS}}(S)=\inf _{\substack{T \text { finite-state } \\ \text { transducer }}} \liminf _{n \rightarrow \infty} \frac{\min _{\pi \in \Sigma^{*}}\{|\pi| \mid T(\pi)=S[0 . . n-1]\}}{n},
$$

and

$$
\operatorname{Dim}_{\mathrm{FS}}(S)=\inf _{\substack{T \text { finite-state } \\ \text { transducer }}} \limsup _{n \rightarrow \infty} \frac{\min _{\pi \in \Sigma^{*}}\{|\pi| \mid T(\pi)=S[0 . . n-1]\}}{n} .
$$

Theorems 3.2 and 3.3 are instances of the existing relation between dimension and information. It is interesting to view them in comparison with other information characterizations of effective dimension that we will develop in the following sections. In the case of constructive dimension, the characterization is based on general Kolmogorov Complexity, which can only be viewed as decompression. For space bounds, dimension can be characterized either by spacebounded compressors or by decompressors, whereas in the case of polynomialtime dimension the known characterization requires to consider polynomial-time 
compressors that are also decompressible in polynomial time. The above results show that finite-state dimension is similar to space-dimension in this matter and apparently simpler than the time-bounded and constructive cases.

We now present a third characterization of finite-state dimension, this time in terms of block-entropy rates.

Definition. Let $w \in \Sigma^{*}, S \in \Sigma^{\infty}$.

1. Let $P(w, S[0 . . k|w|-1])=\frac{1}{k}|\{0 \leq i<k \mid S[i|w| . .(i+1)|w|-1]=w\}|$.

2. The $l$ th block-entropy rate of $S$ is

$$
H_{l}(S)=\liminf _{k \rightarrow \infty}-\frac{1}{l \log |\Sigma|} \sum_{|w|=l} P(w, S[0 . . k l-1]) \log (P(w, S[0 . . k l-1]))
$$

3. The block entropy rate of $S$ is $H(S)=\inf _{l \in \mathbb{N}} H_{l}(S)$.

4. The $l$ th upper block-entropy rate of $S$ is

$$
\overline{H_{l}}(S)=\limsup _{k \rightarrow \infty}-\frac{1}{l \log |\Sigma|} \sum_{|w|=l} P(w, S[0 . . k l-1]) \log (P(w, S[0 . . k l-1]))
$$

5. The upper block-entropy rate of $S$ is $\bar{H}(S)=\inf _{l \in \mathbb{N}} H_{l}(S)$.

Theorem 3.4 [2] Let $S \in \Sigma^{\infty}$. $\operatorname{Dim}_{\mathrm{FS}}(S)=\bar{H}(S)$, and $\operatorname{dim}_{\mathrm{FS}}(S)=H(S)$.

The first part of Theorem 3.4 follows from [37] and [1].

We can also consider "sliding window" entropy, based on the number of times each string $w \in \Sigma^{*}$ appears inside an infinite sequence $S \in \Sigma^{\infty}$ when occurrences can partially overlap.

Definition. Let $w \in \Sigma^{*}, S \in \Sigma^{\infty}$.

1. Let $P^{\prime}(w, S[0 . . n-1])=\frac{|w|}{n}|\{0 \leq i \leq n-|w| \mid S[i . . i+|w|-1]=w\}|$.

2. The $l$ th entropy rate of $S$ is

$$
H_{l}^{\prime}(S)=\liminf _{n \rightarrow \infty}-\frac{1}{l \log |\Sigma|} \sum_{|w|=l} P^{\prime}(w, S[0 . . n-1]) \log \left(P^{\prime}(w, S[0 . . n-1])\right)
$$

3. The entropy rate of $S$ is $H^{\prime}(S)=\inf _{l \in \mathbb{N}} H_{l}^{\prime}(S)$.

4. The $l$ th upper entropy rate of $S$ is

$$
\overline{H_{l}^{\prime}}(S)=\limsup _{n \rightarrow \infty}-\frac{1}{l \log |\Sigma|} \sum_{|w|=l} P^{\prime}(w, S[0 . . n-1]) \log \left(P^{\prime}(w, S[0 . . n-1])\right)
$$

5. The upper entropy rate of $S$ is $\overline{H^{\prime}}(S)=\inf _{l \in \mathbb{N}} H_{l}^{\prime}(S)$. 
The following characterization follows from the results in [37] and Theorem 3.4 .

Theorem 3.5 Let $S \in \Sigma^{\infty}$. $\operatorname{Dim}_{\mathrm{FS}}(S)=\overline{H^{\prime}}(S)$, and $\operatorname{dim}_{\mathrm{FS}}(S)=H^{\prime}(S)$.

Notice that the definitions of entropy consider only frequency properties of the sequence and do not involve finite-state machines, i.e. finite-state dimension admits a "machine independent" characterization.

As a consequence of Theorem 3.4 and previous results in [6], the sequences that have finite-state dimension 1 are exactly the (Borel) normal sequences. Therefore finite-state dimension is base dependent.

Theorem 3.6 There exists a real number $\alpha \in[0,1]$ and $n, m \in \mathbb{N}$ such that the sequences $S$ and $S^{\prime}$ that represent $\alpha$ in bases $n$ and $m$, respectively, have different finite-state dimensions.

The proof of this last theorem is based on the existence of normal sequences that are not absolutely normal, that is, existence of a real number $\alpha$ and two bases $n, m$ such that the representation of $\alpha$ in base $n$ is a normal sequence whereas the representation in base $m$ is not normal (proven by Cassels in [5]).

Hausdorff and packing dimension are both base independent and it is known [33] that polynomial-time dimension is also base independent.

We conclude this section with a summary of the other results on finite-state dimension.

The theorem of Eggleston [14] (Theorem 2.9) holds for finite-state dimension.

Theorem 3.7 [6] For all $\alpha \in \mathbb{Q} \cap[0,1]$,

$$
\operatorname{dim}_{\mathrm{FS}}(\operatorname{FREQ}(\alpha))=\mathcal{H}(\alpha) .
$$

The following theorem says that every rational number $r \in[0,1]$ is the finitestate dimension of a reasonably simple sequence.

Theorem 3.8 [6] For every $r \in \mathbb{Q} \cap[0,1]$ there exists $S \in \mathrm{AC}_{0}$ such that $\operatorname{dim}_{\mathrm{FS}}(S)=r$.

Doty et al. prove that finite-state dimension is invariant under arithmetical operations with a rational number.

Theorem 3.9 [9] Let $k \in \mathbb{N}, q \in \mathbb{Q}$ with $q \neq 0, \alpha \in \mathbb{R}$. Then

$$
\operatorname{dim}_{\mathrm{FS}}\left(S_{q+\alpha}\right)=\operatorname{dim}_{\mathrm{FS}}\left(S_{q \alpha}\right)=\operatorname{dim}_{\mathrm{FS}}\left(S_{\alpha}\right)
$$

where $S_{x}$ is the representation of $x$ in base $k$. The same result holds for Dim $\mathrm{FS}_{\mathrm{FS}}$ in the place of $\operatorname{dim}_{\mathrm{FS}}$.

Scaled-dimension has not been used in the context of finite-state dimension. Notice that only scales of the form $x^{f(s)}$ are finite-state-computable.

Finite-state dimension is a real-time effectivization of a powerful tool of fractal geometry. As such it should prove to be a useful tool for improving our understanding of real-time information processing. 


\section{Constructive Dimension}

Our next effective version of Hausdorff dimension is defined by restricting the class of gales to those that are lower semicomputable. We give the definitions of constructive dimension and constructive strong dimension of a set, and also of a sequence, and we relate them and give their main properties, that make it very powerful. We first have absolute stability, which means it can be applied to an arbitrary union of sets. Then there is a precise characterization of the dimension of a sequence in terms of the Kolmogorov complexity of its elements, and finally in many interesting cases constructive dimension coincides with classical Hausdorff dimension. We also summarize the known relationships of this concept with Martin-Löf random sequences.

An $s$-gale $d$ is constructive if it is lower semicomputable, that is, its lower graph $\{(w, z) \mid z<d(w)\}$ is c.e. We define constructive dimension as follows. Definition. $[46,1]$ Let $X \subseteq \Sigma^{\infty}$.

1. The constructive dimension of a set $X \subseteq \Sigma^{\infty}$ is $\operatorname{cdim}(X)=\inf \left\{s \in[0, \infty) \mid\right.$ there is a constructive $s$-gale $d$ with $\left.X \subseteq S^{\infty}[d]\right\}$.

2. The constructive strong dimension of a set $X \subseteq \Sigma^{\infty}$ is $\operatorname{cDim}(X)=\inf \left\{s \in[0, \infty) \mid\right.$ there is a constructive $s$-gale $d$ with $\left.X \subseteq S_{\text {str }}^{\infty}[d]\right\}$.

3. The (constructive) dimension and strong dimension of an individual sequence $S \in \Sigma^{\infty}$ are $\operatorname{dim}(S)=\operatorname{cdim}(\{S\})$ and $\operatorname{Dim}(S)=\operatorname{cDim}(\{S\})$.

By the gale characterizations of Hausdorff dimension (Theorem 2.8), we conclude that $\operatorname{cdim}(X) \geq \operatorname{dim}_{\mathrm{H}}(X)$ for all $X \subseteq \Sigma^{\infty}$. But in fact much more is true for certain classes, as Hitchcock shows in [24]. For sets that are low in the arithmetical hierarchy, constructive dimension and Hausdorff dimension coincide.

Theorem 4.1 [24] If $X \subseteq \Sigma^{\infty}$ is a union of $\Pi_{1}^{0}$ sets, then $\operatorname{dim}_{\mathrm{H}}(X)=\operatorname{cdim}(X)$.

Hitchcock also proves that this is an optimal result for the arithmetical hierarchy, since it cannot be extended to sets in $\Pi_{2}^{0}$. It is open whether such a correspondence principle holds for strong constructive dimension and packing dimension.

For Hausdorff dimension, all singletons have dimension 0 and in fact all countable sets have Hausdorff dimension 0. The situation changes dramatically when we restrict to constructive gales, since a singleton can have positive constructive dimension, and in fact can have any constructive dimension.

Theorem 4.2 [46] For every $\alpha \in[0,1]$, there is an $S \in \Sigma^{\infty}$ such that $\operatorname{dim}(S)=$ $\alpha$.

A sequence is c-regular if its (constructive) dimension and strong dimensions coincide. In fact these two dimensions can have any arbitrary two values. 
Theorem 4.3 [1] For every $\alpha, \beta \in[0,1]$ with $\alpha \leq \beta$, there is an $S \in \Sigma^{\infty}$ such that $\operatorname{dim}(S)=\alpha$ and $\operatorname{Dim}(S)=\beta$.

An interesting example of a c-regular sequence is $\theta_{A}^{s}$ that generalizes Chaitin's $\Omega$ and has been defined by Tadaki [72] and Mayordomo [50]. $\theta_{A}^{s}$ has dimension and strong dimension $s$.

The constructive dimension of any set $X \subseteq \Sigma^{\infty}$ is completely determined by the dimension of the individual sequences in the set.

Theorem $4.4[46,1]$ For all $X \subseteq \Sigma^{\infty}$,

$$
\operatorname{cdim}(X)=\sup _{x \in X} \operatorname{dim}(x),
$$

and

$$
\operatorname{cDim}(X)=\sup _{x \in X} \operatorname{Dim}(x) .
$$

There is no analogue of this last theorem for Hausdorff dimension or for any of the concepts defined in sections 3 and 5. The key ingredient in the proof of Theorem 4.4 is the existence of optimal constructive supergales, that is, constructive supergales that multiplicatively dominate any other constructive supergale. This is analogous to the existence of universal tests of randomness in the theory of random sequences.

Theorem 4.1 together with Theorem 4.4 implies that the classical Hausdorff dimension of every $\Sigma_{2}^{0}$ set $X \subseteq \Sigma^{\infty}$ has the pointwise characterization $\operatorname{dim}_{H}(X)=\sup _{x \in X} \operatorname{dim}(x)$.

Theorem 4.4 immediately implies that constructive and strong constructive dimensions have the absolute stability property. Classical Hausdorff and packing dimensions have only countable stability.

Corollary $4.5[46,1]$ For any I

$$
\begin{aligned}
& \operatorname{cdim}\left(\bigcup_{i \in I} X_{i}\right)=\sup _{i \in I} \operatorname{cdim}\left(X_{i}\right) . \\
& \mathrm{cDim}\left(\bigcup_{i \in I} X_{i}\right)=\sup _{i \in I} \operatorname{cDim}\left(X_{i}\right) .
\end{aligned}
$$

The (constructive) dimension of a sequence can be characterized in terms of the Kolmogorov complexities of its prefixes. Notice that Kolmogorov complexity is defined as the shortest binary description.

Theorem 4.6 ([50]) For all $A \in \Sigma^{\infty}$,

$$
\operatorname{dim}(A)=\liminf _{n \rightarrow \infty} \frac{\mathrm{K}(A[0 . . n-1])}{n \log |\Sigma|}
$$


This latest theorem justifies the intuition that the constructive dimension of a sequence is a measure of its algorithmic information density. Several authors have studied the close relation of Hausdorff dimension to measures of information content. Ryabko [57, 58], Staiger [68, 69], and Cai and Hartmanis [3] proved results relating Hausdorff dimension to Kolmogorov complexity. Ryabko [60] and Staiger [69] studied computable exponents of increase, that correspond to computable dimension [45], defined in terms of computable gales and that is strictly above constructive dimension. See [46] for a complete chronology.

We note that Theorem 4.6 yields a new proof of Theorem 4.1 above from Theorem 5 of Staiger [69]. Also, Theorem 4.6 yields a new proof of Theorem 4.2 below from Lemma 3.4 of Cai Hartmanis [3].

A dual result holds for constructive strong dimension as proven in [1], that is, for any $A \in \Sigma^{\infty}$,

$$
\operatorname{Dim}(A)=\limsup _{n \rightarrow \infty} \frac{\mathrm{K}(A[0 . . n-1])}{n \log |\Sigma|} .
$$

Alternative characterizations of constructive dimension in terms of variations of Martin-Löf tests and effectivizations of Hausdorff measure have been given by Reimann and Stephan [54] and Calude et al. [4]. Doty has considered Turing reduction compression ratio in [8].

We now briefly state the main results proven so far on constructive dimension, including the existence of sequences of any dimension, the constructive version of Eggleston theorem, and the constructive dimension of sequences that are random relative to a non-uniform distribution.

This is the constructive version of the classical Theorem 2.9 (Eggleston [14]).

Theorem 4.7 [46] If $\alpha$ is $\Delta_{2}^{0}$-computable real number in $[0,1]$ then

$$
\operatorname{cdim}(\operatorname{FREQ}(\alpha))=\mathcal{H}(\alpha) .
$$

An alternative proof of Theorem 4.7 can be derived from Theorem 4.6 and earlier results of Eggleston [14] and Kolmogorov [75]. In fact, this approach shows that Theorem 4.7 holds for arbitrary $\alpha \in[0,1]$.

A binary sequence is (Martin-Löf) random [48] if it passes every algorithmically implementable test of randomness. This can be reformulated in terms of martingales as follows

Definition. [62] A sequence $A \in\{0,1\}^{\infty}$ is (Martin-Löf) random if there is no constructive martingale $d$ such that $A \in S^{\infty}[d]$.

By definition, random sequences have constructive dimension 1. For nonuniform distributions we have the concept of $\beta$-randomness, for $\beta$ any real number in $(0,1)$ representing the bias.

Definition. [62] Let $\beta \in(0,1)$.

1. A $\beta$-martingale is a function $d:\{0,1\}^{*} \rightarrow[0, \infty)$ that satisfies the condition

$$
d(w)=(1-\beta) d(w 0)+\beta d(w 1)
$$

for all $w \in\{0,1\}^{*}$. 
2. A sequence $A \in\{0,1\}^{\infty}$ is (Martin-Löf) random relative to $\beta$ if there is no constructive $\beta$-martingale $d$ such that $A \in S^{\infty}[d]$.

Lutz relates randomness relative to a non-uniform distribution to Shannon information theory.

Theorem 4.8 [46] Let $\beta \in(0,1)$ be a computable real number. Let $A \in\{0,1\}^{\infty}$ be random relative to $\beta$. Then $\operatorname{dim}(A)=\mathcal{H}(\beta)$.

A more general result for randomness relative to sequences of coin-tosses is obtained in [46], and extended in [1], where constructive and constructive strong dimension of such a random sequence are shown to be the lower and upper average entropy of the bias, respectively.

A very recent line of research is the comparison of positive dimension sequences with (Martin-Löf) random sequences (relative to bias $1 / 2$ ) in terms of their computing power. The main issue is whether positive dimension sequences can substitute random sequences as randomness sources [51]. Doty [7], based on earlier results by Ryabko [57, 58], has proven that a sequence of positive dimension is Turing equivalent to a sequence of strong dimension arbitrarily close to 1. Nies and Reimann [53] and Stephan [70] study the existence of weak-truthtable degrees or lower cones of arbitrary dimension. Gu and Lutz [22] show that positive dimension sequences can substitute randomness in the context of probabilistic polynomial-time computation.

We end this section by going back to scaled-dimension. We think that constructive dimension can benefit specially from the flexibility provided by using different scales.

Let $h$ be a scale such that (i) $h(x, 1)=\Theta(x)$ (ii) $d h: \mathbb{N} \times[0, \infty) \rightarrow[0, \infty)$, $d h(k, s)=h\left(|\Sigma|^{-k-1}, s\right) / h\left(|\Sigma|^{-k}, s\right)$ is a computable function. Given a sequence $S$ we define

$\operatorname{dim}^{(h)}(S)=\inf \left\{s \in[0, \infty) \mid\right.$ there is a constructive $s^{(h)}$-gale $d$ with $\left.S \in S^{\infty}[d]\right\}$.

The results in [32] can be extended as follows.

Theorem 4.9 Let $h$ be a scale as above and such that $h(x, s) \leq(\log (1 / x))^{-1-\epsilon}$ for some epsilon (that may depend on $s$ ). Then the following are equivalent.

1. $\operatorname{dim}^{(h)}(S)<s$

2. $K(S[0 . . n-1])<-\log \left(h\left(|\Sigma|^{-n}, s\right)\right)$ for infinitely many $n$.

There is a strong dimension version of Theorem 4.9 in which the Kolmogorov complexity is bounded for almost every prefix of the sequence. In both cases the upper bound on the scale can be substituted by differentiability of $h$. 


\section{Resource-bounded dimension}

In this section we briefly review the properties of resource-bounded dimension more directly related to algorithmic information theory. For a recent summary of dimension in complexity classes the reader may consult [28].

We will consider polynomial-time and polynomial-space dimensions. We define $\mathrm{p}$ to be the class of polynomial time computable functions, pspace as the class of polynomial space functions. Let $\Delta$ be either $\mathrm{p}$ or pspace.

Definition. [45] Let $X \subseteq \Sigma^{\infty}$.

1. The $\Delta$-dimension of a set $X \subseteq \Sigma^{\infty}$ is $\operatorname{dim}_{\Delta}(X)=\inf \left\{s \in[0, \infty) \mid\right.$ there is a s-gale $d \in \Delta$ with $\left.X \subseteq S^{\infty}[d]\right\}$.

2. The $\Delta$ strong dimension of a set $X \subseteq \Sigma^{\infty}$ is

$\operatorname{Dim}_{\Delta}(X)=\inf \left\{s \in[0, \infty) \mid\right.$ there is a $s$-gale $d \in \Delta$ with $\left.X \subseteq S_{\text {str }}^{\infty}[d]\right\}$.

Let us mention that Eggleston theorem also holds for the resource-bounded case [45], for each p-computable (pspace-computable) $\alpha, \operatorname{dim}_{\mathrm{p}}(\operatorname{FREQ}(\alpha))=$ $\mathcal{H}(\alpha)\left(\operatorname{dim}_{\text {pspace }}(\operatorname{FREQ}(\alpha))=\mathcal{H}(\alpha)\right)$, and even for sublinear time-bounds [52].

Hitchcock [25] has characterized pspace-dimension in terms of space-bounded Kolmogorov complexity as follows. Let $\operatorname{KS}^{f(n)}(w)$ be the Kolmogorov complexity of the string $w$ when only space $f(|w|)$ is allowed in the computation of $w$ from its description [40].

Theorem 5.1 ([25]) For all $X \subseteq \Sigma^{\infty}$,

$$
\begin{gathered}
\operatorname{dim}_{\text {pspace }}(X)=\inf _{c} \sup _{A \in X} \liminf _{n \rightarrow \infty} \frac{\operatorname{KS}^{n^{c}}(A[0 . . n-1])}{n \log |\Sigma|} \\
\operatorname{Dim}_{\text {pspace }}(X)=\inf _{c} \sup _{A \in X} \limsup _{n \rightarrow \infty} \frac{\operatorname{KS}^{n^{c}}(A[0 . . n-1])}{n \log |\Sigma|}
\end{gathered}
$$

This result can also be extended to scaled-dimension.

Theorem 5.2 [32] For $z \in\{-1,0,1\}$ the following are equivalent.

1. $\operatorname{dim}_{\mathrm{pspace}}^{(z)}(X)<s$

2. There exists c such that for every $S \in X$,

$$
\mathrm{KS}^{n^{c}}(S[0 . . n-1])<-\log \left(h_{z}\left(|\Sigma|^{-n}, s\right)\right) \text { for infinitely many } n .
$$

Theorem 5.2 has a strong dimension version in which the Kolmogorov complexity is bounded for almost every prefix of the sequence.

The case of polynomial-time dimension seems much harder, since timebounded Kolmogorov complexity has proven difficult to analyze. After attempts 
from Hitchcock and Vinodchandran in [29], the right approach seems to be the consideration of polynomial time compressors that can also be inverted in polynomial time. López-Valdés and Mayordomo [41] prove the following.

Definition. [41] Let $(C, D)$ be polynomial-time algorithms with input and output alphabet $\Sigma$ and such that for every $w \in \Sigma^{*}, D(C(w),|w|)=w \cdot(C, D)$ does not start from scratch if $\forall \epsilon>0$ and for almost every $w \in \Sigma^{*}$ there exists $k=O(\log (|w|)), k>0$, such that

$$
\sum_{|u| \leq k}|\Sigma|^{-|C(w u)|} \leq|\Sigma|^{\epsilon k}|\Sigma|^{-|C(w)|} .
$$

Let PC be the class of polynomial-time compressors that do not start from scratch.

Theorem 5.3 [41] Let $X \subseteq \Sigma^{\infty}$,

$$
\begin{aligned}
\operatorname{dim}_{\mathrm{p}}(X) & =\inf _{(C, D) \in \mathrm{PC}} \sup _{A \in X} \liminf _{n} \frac{|C(A[0 \ldots n-1])|}{n}, \\
\operatorname{Dim}_{\mathrm{p}}(X) & =\inf _{(C, D) \in \mathrm{PC}} \sup _{A \in X} \limsup _{n} \frac{|C(A[0 \ldots n-1])|}{n} .
\end{aligned}
$$

Connection of resource-bounded dimension with sequence analysis models from computational learning has proven successful in [31], [26] and [20].

Acknowledgments. I thank an anonymous referee, Philippe Moser, and David Doty for many helpful suggestions.

\section{References}

[1] K. B. Athreya, J. M. Hitchcock, J. H. Lutz, and E. Mayordomo. Effective strong dimension in algorithmic information and computational complexity. SIAM Journal on Computing. To appear.

[2] C. Bourke, J. M. Hitchcock, and N. V. Vinodchandran. Entropy rates and finite-state dimension. Theoretical Computer Science, 349:392-406, 2005.

[3] J. Cai and J. Hartmanis. On Hausdorff and topological dimensions of the Kolmogorov complexity of the real line. Journal of Computer and Systems Sciences, 49:605-619, 1994.

[4] C.S. Calude, L. Staiger, and S. A. Terwijn. On partial randomness. Annals of Pure and Applied Logic, 138:20-30, 2006.

[5] J.W.S. Cassels. On a problem of steinhaus about normal numbers. Colloquium Mathematicum, 7:95-101, 1959.

[6] J. J. Dai, J. I. Lathrop, J. H. Lutz, and E. Mayordomo. Finite-state dimension. Theoretical Computer Science, 310:1-33, 2004. 
[7] D. Doty. Dimension extractors. Technical Report Technical Report cs.CC/0606078, Computing Research Repository, 2006.

[8] D. Doty. Every sequence is decompressible from a random one. In Proceedings of Second Conference on Computability in Europe, Lecture Notes in Computer Science, pages 153-162. Springer-Verlag, 2006.

[9] D. Doty, J. H. Lutz, and S. Nandakumar. Finite-state dimension and real arithmetic. In Proceedings of the 33rd International Colloquium on Automata, Languages, and Programming, Lecture Notes n Computer Science. Springer-Verlag, 2006.

[10] D. Doty and P. Moser. Personal communication, based on [67]. 2006.

[11] R. Downey and D. Hirschfeldt. Algorithmic randomness and complexity. Book Draft, 2006.

[12] G. A. Edgar. Integral, probability, and fractal measures. Springer-Verlag, 1998.

[13] G.A. Edgar. Measure, Topology, and Fractal Geometry. Springer-Verlag, 1990.

[14] H.G. Eggleston. The fractional dimension of a set defined by decimal properties. Quarterly Journal of Mathematics, Oxford Series 20:31-36, 1949.

[15] K. Falconer. The Geometry of Fractal Sets. Cambridge University Press, 1985.

[16] K. Falconer. Fractal Geometry: Mathematical Foundations and Applications. John Wiley \& sons, 2003.

[17] K. Falconer. Techniques in Fractal Geometry. John Wiley \& sons, 2003.

[18] M. Feder. Gambling using a finite state machine. IEEE Transactions on Information Theory, 37:1459-1461, 1991.

[19] H Federer. Geometric Measure Theory. Springer-Verlag, 1969.

[20] L. Fortnow and J. H. Lutz. Prediction and dimension. Journal of Computer and System Sciences. To appear. Preliminary version appeared in Proceedings of the 15th Annual Conference on Computational Learning Theory, LNCS 2375, pages 380-395, 2002.

[21] X. Gu, J. H. Lutz, and E. Mayordomo. Points on computable curves. In Proceedings of the Forty-Seventh Annual IEEE Symposium on Foundations of Computer Science, 2006. To appear.

[22] X. Gu and J.H. Lutz. Dimension characterizations of complexity classes. In Proceedings of the 31st International Symposium on Mathematical Foundations of Computer Science, Lecture Notes in Computer Science, pages 471-479. Springer-Verlag, 2006. 
[23] F. Hausdorff. Dimension und äußeres Maß. Math. Ann., 79:157-179, 1919.

[24] J. Hitchcock. Correspondence principles for effective dimensions. In Proceedings of the 29th Colloquium on Automata, Languages and Programming. Springer Lecture Notes in Computer Science, 2002. To appear.

[25] J. M. Hitchcock. Effective Fractal Dimension: Foundations and Applications. PhD thesis, Iowa State University, 2003.

[26] J. M. Hitchcock. Fractal dimension and logarithmic loss unpredictability. Theoretical Computer Science, 304(1-3):431-441, 2003.

[27] J. M. Hitchcock, J. H. Lutz, and E. Mayordomo. Scaled dimension and nonuniform complexity. Journal of Computer and System Sciences, 69:97122,2004 .

[28] J. M. Hitchcock, J. H. Lutz, and E. Mayordomo. The fractal geometry of complexity classes. SIGACT News Complexity Theory Column, 36:24-38, 2005.

[29] J. M. Hitchcock and N. V. Vinodchandran. Dimension, entropy rates, and compression. Journal of Computer and System Sciences, 72(4):760-782, 2006 .

[30] J.M. Hitchcock. Effective fractal dimension bibliography. http://www.cs.uwyo.edu/ jhitchco/bib/dim.shtml.

[31] J.M. Hitchcock. Online learning and resource-bounded dimension: Winnow yields new lower bounds for hard sets. SIAM Journal on Computing, 2006. To appear.

[32] J.M. Hitchcock, M. López-Valdés, and E. Mayordomo. Scaled dimension and the Kolmogorov complexity of Turing-hard sets. In Proceedings of the 29th International Symposium on Mathematical Foundations of Computer Science, volume 3153 of Lecture Notes in Computer Science, pages 476-487. Springer-Verlag, 2004.

[33] J.M. Hitchcock and E. Mayordomo. Base invariance of feasible dimension. Manuscript, 2003.

[34] D. A. Huffman. Canonical forms for information-lossless finite-state logical machines. IRE Trans. Circuit Theory CT-6 (Special Supplement), pages 41-59, 1959. Also available in E.F. Moore (ed.), Sequential Machine: Selected Papers, Addison-Wesley, 1964, pages 866-871.

[35] Z. Kohavi. Switching and Finite Automata Theory (Second Edition). McGraw-Hill, 1978.

[36] A. A. Kurmit. Information-Lossless Automata of Finite Order. Wiley, 1974. 
[37] A. Lempel and J. Ziv. Compression of individual sequences via variable rate coding. IEEE Transaction on Information Theory, 24:530-536, 1978.

[38] P. Lévy. Propriétés asymptotiques des sommes de variables indépendantes ou enchainées. Journal des mathématiques pures et appliquées. Series 9., 14(4):347-402, 1935.

[39] P. Lévy. Théorie de l'Addition des Variables Aleatoires. Gauthier-Villars, 1937 (second edition 1954).

[40] M. Li and P. M. B. Vitányi. An Introduction to Kolmogorov Complexity and its Applications. Springer-Verlag, Berlin, 1997. Second Edition.

[41] M. López-Valdés and E. Mayordomo. Dimension is compression. In Proceedings of the 30th International Symposium on Mathematical Foundations of Computer Science, volume 3618 of Lecture Notes in Computer Science, pages 676-685. Springer-Verlag, 2005.

[42] J. H. Lutz. Almost everywhere high nonuniform complexity. Journal of Computer and System Sciences, 44(2):220-258, 1992.

[43] J. H. Lutz. The quantitative structure of exponential time. In L. A. Hemaspaandra and A. L. Selman, editors, Complexity Theory Retrospective II, pages 225-254. Springer-Verlag, 1997.

[44] J. H. Lutz. Resource-bounded measure. In Proceedings of the 13th IEEE Conference on Computational Complexity, pages 236-248, 1998.

[45] J. H. Lutz. Dimension in complexity classes. SIAM Journal on Computing, 32:1236-1259, 2003.

[46] J. H. Lutz. The dimensions of individual strings and sequences. Information and Computation, 187:49-79, 2003.

[47] J.H. Lutz and E. Mayordomo. Dimensions of points in self-similar fractals. Abstract published in the proceedings of the Third International Conference on Computability and Complexity in Analysis.

[48] P. Martin-Löf. The definition of random sequences. Information and Control, 9:602-619, 1966.

[49] P. Matilla. Geometry of Sets and Measures in Euclidean Spaces: Fractals and rectifiability. Cambridge University Press, 1995.

[50] E. Mayordomo. A Kolmogorov complexity characterization of constructive Hausdorff dimension. Information Processing Letters, 84(1):1-3, 2002.

[51] J.S. Miller and A. Nies. Randomness and computability: open questions. Bulletin of Symbolic Logic, 12:390-410, 2006. 
[52] P. Moser. Martingale families and dimension in P. In Logical Approaches to Computational Barriers, Second Conference on Computability in Europe, CiE 2006, volume 3988 of Lecture Notes in Computer Science, pages 388397. Springer-Verlag, 2006.

[53] A. Nies and J. Reimann. A lower cone in the wtt degrees of non-integral effective dimension. In Proceedings of IMS workshop on Computational Prospects of Infinity, 2006. To appear.

[54] J. Reimann and F. Stephan. Effective hausdorff dimension. In Logic Colloquium '01, number 20 in Lecture Notes in Logic, pages 369-385. Association for Symbolic Logic, 2005.

[55] J. Reimann and F. Stephan. On hierarchies of randomness tests. In Proceedings of the 9th Asian Logic Conference 2005. World Scientific, 2006.

[56] C. A. Rogers. Hausdorff Measures. Cambridge University Press, 1998. Originally published in 1970.

[57] B. Ya. Ryabko. Coding of combinatorial sources and hausdorff dimension. Soviets Mathematics Doklady, 30:219-222, 1984.

[58] B. Ya. Ryabko. Noiseless coding of combinatorial sources. Problems of Information Transmission, 22:170-179, 1986.

[59] B. Ya. Ryabko. Algorithmic approach to the prediction problem. Problems of Information Transmission, 29:186-193, 1993.

[60] B. Ya. Ryabko. The complexity and effectiveness of prediction problems. Journal of Complexity, 10:281-295, 1994.

[61] C. P. Schnorr. Klassifikation der Zufallsgesetze nach Komplexität und Ordnung. Z. Wahrscheinlichkeitstheorie verw. Geb., 16:1-21, 1970.

[62] C. P. Schnorr. A unified approach to the definition of random sequences. Mathematical Systems Theory, 5:246-258, 1971.

[63] C. P. Schnorr. Zufälligkeit und Wahrscheinlichkeit. Lecture Notes in Mathematics, 218, 1971.

[64] C. P. Schnorr. Process complexity and effective random tests. Journal of Computer and System Sciences, 7:376-388, 1973.

[65] C. P. Schnorr. A survey of the theory of random sequences. In R. E. Butts and J. Hintikka, editors, Basic Problems in Methodology and Linguistics, pages 193-210. D. Reidel, 1977.

[66] C. P. Schnorr and H. Stimm. Endliche automaten und zufallsfolgen. Acta Informatica, 1:345-359, 1972. 
[67] D. Sheinwald, A. Lempel, and J. Ziv. On compression with two-way head machines. In Data Compression Conference, pages 218-227, 1991.

[68] L. Staiger. Kolmogorov complexity and Hausdorff dimension. Information and Computation, 103:159-94, 1993.

[69] L. Staiger. A tight upper bound on Kolmogorov complexity and uniformly optimal prediction. Theory of Computing Systems, 31:215-29, 1998.

[70] F. Stephan. Hausdorff-dimension and weak truth-table reducibility. Technical Report Technical Report TR52/05, National University of Singapore, School of Computing, 2005.

[71] D. Sullivan. Entropy, Hausdorff measures old and new, and limit sets of geometrically finite Kleinian groups. Acta Mathematica, 153:259-277, 1984.

[72] K. Tadaki. A generalization of Chaitin's halting probability $\omega$ and halting self-similar sets. Hokkaido Mathematical Journal, 31:219-253, 2002.

[73] C. Tricot. Two definitions of fractional dimension. Mathematical Proceedings of the Cambridge Philosophical Society, 91:57-74, 1982.

[74] J. Ville. Étude Critique de la Notion de Collectif. Gauthier-Villars, Paris, 1939.

[75] A. K. Zvonkin and L. A. Levin. The complexity of finite objects and the development of the concepts of information and randomness by means of the theory of algorithms. Russian Mathematical Surveys, 25:83-124, 1970. 